\title{
ARTICLE \\ Investigation of adult immunodeficiency and indications for immunoglobulin replacement therapy
}

\author{
S Ress, MB ChB, FCP (SA) \\ Department of Medicine, Faculty of Health Sciences, University of Cape Town, and University of Cape Town Private Academic Hospital, \\ Cape Town, South Africa
}

Corresponding author: S Ress (stan.ress@uct.ac.za)

\begin{abstract}
Adults presenting with recurrent infections require an approach that allows exclusion of underlying immunodeficiency. While secondary causes of immunodeficiency are the most common, primary immunodeficiency disease (PID) may present for the first time in adults. Failure to consider this diagnosis in adults and children leads to a major diagnostic delay. Recurrent localised infections generally suggest an underlying anatomical rather than an immune defect. PIDs that are most commonly encountered include common variable immunodeficiency, immunoglobulin (Ig) A deficiency, and IgG subclass deficiency. I suggest a diagnostic approach with relevant immune tests, depending on the clinical picture. The essential role of vaccination with polysaccharide and protein antigens to evaluate B-cell functional capacity, is highlighted. Principles of management are provided, including indications for immunoglobulin replacement therapy.
\end{abstract}

S Afr Med J 2014;104(11):791-792. DOI:10.7196/SAMJ.8948

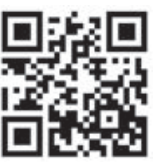

It is not uncommon for a clinician to be faced with a patient who presents with recurrent infections. This leads to the question whether there is an underlying immunodeficiency. Adult patients with recurrent infections may fall into one of three groups:

- Patients presenting with recurrent localised infections: it is mandatory to exclude an underlying predisposing, regional anatomical defect.

- Patients with secondary immunodeficiency: it can be caused by an underlying disease or a complication of immune suppression (Table 1).

- A wide variety of primary immunodeficiency diseases (PIDs) presenting in childhood can persist into adulthood, while rare disorders such as cystic fibrosis and chronic granulomatous disease (CGD) can present for the first time in adults. Primary immune disorders can also present for the first time in adulthood and the conditions most likely to be encountered are discussed briefly below. Autoimmunity and malignancy are commonly associated with PID because of the defective immunity associated with these conditions.

\section{Defects in humoral immunity \\ Selective IgA deficiency}

There may be a partial reduction or a severe deficiency of serum immunoglobulin (Ig) A. ${ }^{[1]}$
Patients may be asymptomatic or present with recurrent sinopulmonary infections, especially when associated with IgG subclass deficiency, and gastrointestinal (GIT) infections such as recurrent giardiasis. Drugs such as anticonvulsants and sulfasalazine may also cause $\operatorname{IgA}$ deficiency, which may be reversible on discontinuation of the medication. There is a higher incidence of allergic rhinitis, asthma, and autoimmune disorders, including cytopenias and systemic lupus erythematosus (SLE), and some patients may develop common variable immunodeficiency (CVID). Patients with severe IgA deficiency are at risk for anaphylactic transfusion reactions as a result of anti-IgA antibodies in their serum reacting with transfused IgA components.

\begin{abstract}
Abnormalities in serum IgM levels
Selective IgM deficiency is rare and can be an incidental finding in otherwise asymptomatic patients, or patients may present with recurrent respiratory infections with polysaccharide-containing organisms such as pneumococci and Haemophilus influenzae..$^{[2,3}$ Asthma and allergic rhinitis are commonly associated, while fibromyalgia-like symptoms and autoimmunity also occur. Patients shown to have antigen-specific defects can benefit from immunoglobulin replacement.

Patients with hyper-IgM usually present in childhood with PID - with elevated serum
\end{abstract}

\section{Table 1. Acquired, secondary causes of immunodeficiency}
- HIV infection
- Post-splenectomy
- Diabetes
- Chronic renal disease - especially nephrotic syndrome
- Chronic liver cirrhosis
- Haematological disorders: chronic lymphocytic leukaemia, myeloma
- Immune suppression due to radiation, cytotoxic and/or steroid therapy
- Malnutrition, vitamin and mineral deficiency (e.g. zinc deficiency)
- Autoimmune disorders, e.g systemic lupus erythematosus
- Intestinal lymphangiectasia resulting in immunoglobulin and lymphocyte loss
- Malignancy, including thymoma with hypogammaglobulinaemia - Good's syndrome 
IgM but reduced IgG and IgA levels. ${ }^{[4]}$ This is a genetically heterogeneous disorder but the essential defect is in CD40-mediated signalling, causing defective monocyte, B-cell and T-cell function. This results in recurrent bacterial and opportunistic infections due to Pneumocystis and Cryptosporidium organisms. Milder mutations rarely result in a less severe clinical course that may present later in life. ${ }^{[4]}$

Common variable immunodeficiency CVID is the commonest primary, intrinsic disorder of antibody production in both children and adults. It is defined by a markedly reduced serum IgG with low IgA and/or IgM together with: (i) proven lack of specific $\operatorname{IgG}$ production following test immunisations; and (ii) exclusion of B-cell defects (e.g. IgG subclass deficiency) and haematological conditions (e.g. chronic lymphocytic leukaemia (CLL) and myeloma). ${ }^{[5]}$

CVID is a heterogeneous, clinically diverse disorder. Recurrent sinusitis, upper respiratory tract infections and pneumonia can lead to bronchiectasis. Complications include autoimmune cytopenias, enteropathy, and lymphoid malignancies. ${ }^{[6]}$ Liver disease occurs; the most common finding is raised alkaline phosphatase due to nodular regenerative hyperplasia, which is commonly associated with idiopathic non-cirrhotic portal hypertension. ${ }^{[7,8]}$ Hepatitis B and C infections, primary biliary cirrhosis, granulomatous liver disease and autoimmune-like liver disease are also found. Immunoglobulin replacement therapy protects against infection but does not prevent the other complications. ${ }^{[6]}$

\section{IgG subclass deficiency and specific antibody deficiency}

Clinically significant IgG subclass deficiency requires a significant reduction in one or more IgG subclass concentrations, together with evidence of antibody dysfunction evidenced by recurrent infections, and an inadequate response to vaccine challenge. ${ }^{[9]}$ Recurrent infections may occur despite normal total serum immunoglobulin and IgG subclass levels owing to specific antibody deficiency (SAD). This is confirmed by failure to respond to test vaccines. If sinopulmonary infections persist despite aggressive treatment of predisposing allergic rhinitis and asthma and prophylactic antibiotics, then immunoglobulin replacement therapy may be required in both IgG subclass deficiency and SAD.

\section{Selective IgG deficiency}

Patients with recurrent infections and borderline serum $\operatorname{IgG}$ are commonly referred for immune evaluation. They may have allergic rhinitis and recurrent sinusitis, and are also often asthmatic, requiring significant steroid use. Chronic infections are usually poorly defined and accompanied by fatigue and a modest reduction in serum $\mathrm{IgG}$ of $5-7 \mathrm{~g} / \mathrm{L}$ (normal range $7-16 \mathrm{~g} / \mathrm{L}$ ). Hypogammaglobulinaemia due to steroid use can be diagnosed by preservation of functional antibody production in the form of adequate baseline memory antibodies or a good response to test vaccinations.

\section{Defects in cellular immunity}

Adult patients present far less commonly with primary defects in cellular immunity. Defective cellular immunity often accompanies hypogammaglobulinaemia in CVID. Patients with acquired antibodies to inteferon (IFN)- $\gamma$ are increasingly described ${ }^{[10]}$ and defects in the interleukin (IL)-12 or IFN- type 1 cytokine pathway can present with disseminated Mycobacterium avium or Salmonella infections. ${ }^{[1]}$

\section{Defects in phagocytosis}

These have recently been reviewed ${ }^{[12]}$ and are usually diagnosed in childhood cases of recurrent pyogenic infections, deep-seated abscesses and sepsis, but can also present in adults.

\section{Immune investigations}

Table 2 shows the clinical presentations due to defects in the five main components of protective immunity, which can guide the clinician to the required diagnostic tests. Recurrent localised infections are invariably caused by defects in anatomical barriers, leading to chronic infections. Examples include base of skull fractures presenting with recurrent pneumococcal meningitis, urinary tract obstruction presenting with recurrent urinary infections, and recurrent sinusitis following previous extensive surgery that results in bacterial colonisation on abnormal mucosal surfaces.

Defects in innate immunity can cause recurrent infections; the most commonly encountered example is recurrent viral infection (especially herpes) due to deficiency of natural killer (NK) cells. ${ }^{[13]}$ Patients with deficiency of terminal complement components C7 - C9 present with recurrent Neisseria meningitidis meningitis. Defects in T-cell function present with typical opportunistic organisms, and fungal, parasitic and mycobacterial infections. Defects in phagocytic function can present with infections caused by non-pathogenic bacteria or fungi. Defects in antibody production present with recurrent upper respiratory, sinus and middle-ear infections; these are most commonly encountered in clinical practice.

Quantification of the major immunoglobulin classes IgA, IgM, IgG and $\operatorname{IgE}$ is routinely requested as the initial investigation in cases of suspected humoral immunodeficiency. Where panhypogammaglobulinaemia is documented, myeloma needs to be excluded with serum protein electrophoresis, free serum lightchain assay, and urine light chains. Where immunoglobulin levels are low/normal and/ or an IgG subclass deficiency is suspected, it is customary to request IgG subclasses, but minor reductions below the laboratory normal values are frequently misinterpreted as significant. For a clinically relevant IgG subclass deficiency there needs to be significant reduction in values and it is preferable to consider the subclass percentage distribution rather than absolute values. As a general rule, IgG $1>60 \%, \operatorname{IgG}_{2}>10-15 \%, \mathrm{IgG}_{3}$ $>5 \%$, while $\operatorname{IgG}_{4}$ can be absent in a significant number of normal people. Functional capacity of humoral immunity is also more valuable than serum $\operatorname{IgG}$ quantification; therefore a baseline vaccination status is obtained by requesting serum IgG 'memory'specific antibody levels against capsular polysaccharides of pneumococcus and

Table 2. Clinical picture associated with defective function of specific compartments of the immune system

\begin{tabular}{ll}
\hline Defective immune function & Clinical picture \\
\hline B cell & Recurrent upper respiratory tract infection/pneumonia \\
T cell & Viral/protozoal/fungal/mycobacterial infection \\
Natural killer cell & Recurrent viral \\
Phagocytosis & Supurative skin or systemic infection \\
Complement & Infections/autoimmunity \\
Anatomical defect & Localised infections \\
NB: Recurrent localised infections are generally associated with an anatomical abnormality, and not defective immunity.
\end{tabular}




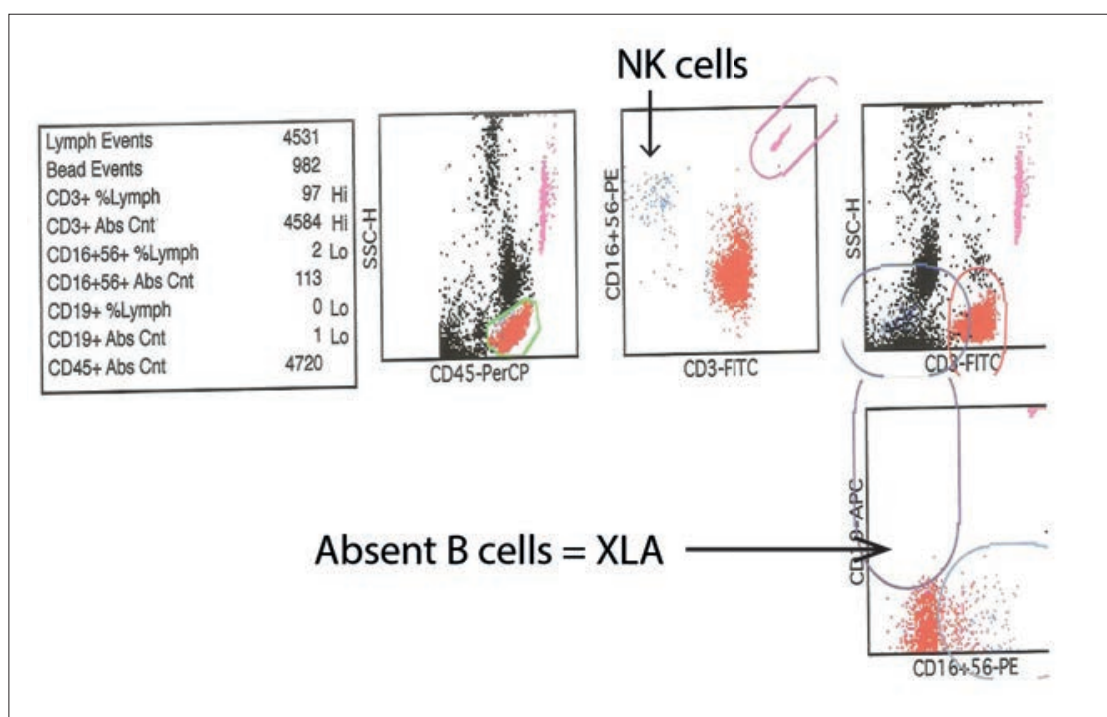

Fig. 1. Flow cytometry demonstrating total absence of B cells in a 16-year-old adolescent who presented with severe necrotising pneumonia and extremely low serum immunoglobulins owing to X-linked agammaglobulinaemia. $\mathrm{CD}^{+}$identifies T cells; $C D 16^{+} 56^{+}$identifies natural killer cells; $C D 19^{+}$identifies $B$ cells; and $C D 45^{+}$identifies all leukocytes and is used to 'gate' on the lymphocyte population. (CD = clusters of differentiation; Abs Cnt = absolute counts; SSC-H = side scatter, as indication of cell granular content, $\mathrm{NK}$ cells $=$ natural killer cells; XLA =X-linked agammaglobulinaemia.)

H. influenzae $\mathrm{B}$, and against tetanus toxoid and diphtheria as representative of protein antigens. If these values are low, the response to vaccination with pneumococcus and tetanus-conjugated $H$. influenzae B (e.g. Hiberix vaccine) is evaluated after $3-4$ weeks. If defects in phagocytic function are suspected, this can be evaluated by flow cytometry to measure phagocytic index and respiratory burst of polymorph neutrophils and monocytes. A full evaluation includes assessment of bacterial killing and leukocyte chemotaxis. Measurement of peripheral blood subsets provides information on the percentage and absolute numbers of circulating T cell, B cell and NK cells, and is currently performed as a single platform by flow cytometry (Fig. 1). Where CVID is suspected, measurement of memory B-cell subsets is of value, as a reduction in 'switched' memory B cells correlates with recurrent respiratory infections and bronchiectasis, ${ }^{[14]}$ more than actual serum immunoglobulin concentration. ${ }^{[15]}$ T-cell function can be assessed by measuring proliferative responses to stimulation by mitogens (phytohaemagglutinin/Con-A or phorbal ester/ionomycin), antigens (purified protein derivative) and Candida. Where proliferative responses are normal and an inhibitor of IFN- $\gamma$ is suspected, such as in disseminated avium infection in an otherwise normal individual, it is imperative to specifically test for a serum autoantibody against IFN- $\gamma \cdot{ }^{[10]}$

\section{Management}

General principles for the management of patients with recurrent infections include:

- Optimal management of any predisposing conditions such as allergic rhinitis and asthma, including allergy evaluation and trigger avoidance, and use of nasal and inhaled steroids.

- Prompt diagnosis and antibiotic therapy of sinopulmonary infections to prevent chronic lung damage from recurrent infections, which can result in bronchiectasis. Bacterial infections should be confirmed, where possible, by complete blood counts, C-reactive protein levels and culturing.

- Patients with poor memory polysaccharide antibody responses should receive vaccination with conjugated 13 -valent pneumococcal vaccine (Prevnar).

- If repeated infections occur despite the above measures a six-month trial of prophylactic antibiotics should be considered.

- In patients with normal or low-normal immunoglobulin levels with recurrent infections, immunoglobulin replacement therapy (subcutaneous (SC) or intravenous (IV) infusion) is generally reserved for those with demonstrated suboptimal responses to vaccination with polysaccharide and protein antigens ${ }^{[16]}$ and failed antibiotic prophylaxis, as treatment is costly, can be associated with side-effects, and is usually lifelong.

- Patients with CVID need weekly SC or monthly intravenous immunoglobulin replacement. They may suffer from giardiasis due to $\operatorname{Ig} A$ deficiency, which cannot be replaced, and resultant malabsorption. They are best managed by a multidisciplinary team consisting of a clinical immunologist, pulmonologist and gastroenterologist.

\section{References}

1. Yel L. Selective IgA Deficiency. J Clin Immunol 2010;30:10-16 [http://dx.doi.org/10.1007/s10875-009-9357-x]

2. Yel L, Ramanuja S, Gupta S. Clinical and immunological feature in IgM deficiency. Int Arch Allergy Immunol 2009;150:291-298 http://dx.doi.org/10.1159/000222682

3. Goldstein MF, Goldstein AL, Dunsky EH, et al. Selective IgM immunodeficiency: Retrospective analysis of 36 adult patients with review of the literature. Ann Allergy Asthm Immunol 2006;97:717-730. [http://dx.doi.org/10.1016/S10811206(10)60962-3]

4. Notarangelo LD, Lanzi G, Peron S, Durandy A. Defects of classswitch recombination. J Allergy Clin Immunol 2006;117:855864. [http://dx.doi.org/10.1016/j.jaci.2006.01.043]

5. Cunningham-Rundles $\mathrm{C}$. How I treat common variable immune deficiency. Blood 2010;116:7-15. [http://dx.doi.org/10.1182 blood-2010-01-254417]

6. Resnick ES, Moshier EL, Godbold JH, Cunningham-Rundles C. Morbidity and mortality in common variable immun deficiency over 4 decades. Blood 2012;119:1650-1657. [http: $/$ dx.doi.org/10.1182/blood-2011-09-377945

7. Ward C, Lucas M, Piris J, et al. Abnormal liver function in common variable immune deficiency disorders due to nodular regenerative hyperplasia. Clin Exp Immunol 2008;153:331-357. [http://dx.doi.org/10.1111/j.1365-2249.2008.03711.x]

8. Fuss IJ, Friend J, Yang $Z$, et al. Nodular regenerative hyperplasia in common variable immunodeficiency. I Clin Immunol 2013;33(4):748-758. [http://dx.doi.org/10.1007/s10875013-9873-6]

9. O'Connor ME, Kirkpatrick CH. Management of IgG subclass deficiency in adults. Infect Med 2003;20:596-602.

0. Kampmann B, Hemingway C, Stephens A, et al. Acquired predisposition to mycobacterial disease due to autoantibodies to IFN- $\gamma$. J Clin Invest 2005;115:2480-2488. [http://dx.doi.org/10.1172/ JCI19316]

11. Lammas DA, Drysdale P, Ben-Smith A, et al. Diagnosis of defects in the type 1 cytokine pathway. Microbes Infect 2000;2:1567 1578. [http://dx.doi.org/10.1016/S1286-4579(00)01313-7]

12. Wolach $B$, Gavrieli R, Roos D, Berger-Achituv S. Lessons learned from phagocytic function studies in a large cohort of patients with recurrent infections. J Clin Immunol 2012;32:454-466. [http://dx.doi.org/10.1007/s10875-011-9633-4]

13. Orange JS. Human natural killer cell deficiencies and susceptibility to infection. Microbes Infect 2002;2:1545-1558.

14. Vodjgani M, Aghamohammadi A, Samadi M, et al. Analysis of class-switched memory B cells in patients with common variable Clin Immunol 2007;17:321-328.

15. Alachkar H, Taubenheim N, Haeney MR. Memory switched B cell percentane and not serum ime is assocented with clinical complications in children and dults with secife antibody deficiency and con dildren and

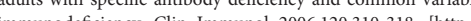
(200605003)

6. Orange JS, Ballow M, Stiehm ER, et al. Use and interpretation of diagnostic vaccination in primary immunodeficiency: working group report of the Basic and Crical lmmunology Interest Section of the American Academy of Allergy, Asthm [htp:/10 